Results: TAB specimens from patients with biopsy-proven GCA, biopsy-negative GCA and controls were positive for anti-IL- 6 staining in $59 \%, 13 \%$ and $48 \%$ of cases, respectively, the difference between biopsy-proven and biopsy-negative GCA patients being significant $(p=0.04)$. In non-inflamed TABs, IL- 6 was mainly expressed by mesenchymal cells in media and intima layers, while in inflamed TABs IL-6 was mainly expressed by mononuclear inflammatory infiltrating cells. IL-6 grade 2-3 expression was observed in all 6 patients with visual loss compared to $25(43.9 \%)$ of 57 patients without $(p=0.011)$. Blindness was recorded in 2 patients with biopsy-proven GCA and 4 controls (all with a final diagnosis of nonarteritic ischaemic optic neuropathy). No associations were found between IL-6 expression and demographic characteristics, GCA signs/symptoms, laboratory and histopathological $T A B$ findings. However, there was a statistical trend $(p=0.055)$ of increased frequency of the halo sign at temporal artery CDS in patients with IL- 6 expression grade $2-3$ compared to those with IL- 6 expression grade $0-1$. No significant differences for the expression of IL-6 were observed between patients with and without PMR (5/8\%-62.5\% - versus $6 / 15 \%-40 \%-$, $\mathrm{p}=0.400$ ) and between patients with isolated PMR and those with TAB positive GCA $(62.5 \%$ vs $59 \%, p=1.000)$

Conclusions: Our study provides evidence that IL-6 expression does not increase the sensitivity of $T A B$ in patients with morphologically uninflamed arteries. A search for further markers that may increase the sensitivity of TAB is warranted.

Disclosure of Interest: None declared

DOI: 10.1136/annrheumdis-2018-eular.6729

\section{THU0458 INVESTIGATION OF THE ROLE OF M-TOR PATHWAY IN KIDNEY NEEDLE BIOPSIES OF PATIENTS WITH ANTINEUTROPHIL CYTOPLASMIC AUTOANTIBODY- ASSOCIATED VASCULITIS}

Z. Soypacaci ${ }^{1}$, O. Cakmak ${ }^{2}$, F. Cakalagaoglu ${ }^{3}$, O. Gercik ${ }^{4}$, I. Ertekin ${ }^{5}$, A. Uzum ${ }^{1}$, R. Ersoy ${ }^{1}$, S. Akar ${ }^{4} .{ }^{1}$ Nephrology, ${ }^{2}$ Internal Medicine, Izmir Katip Celebi University School of Medicine; ${ }^{3}$ Pathology, Izmir Katip Celebi University School of Medicine Ataturk Research Hospital; ${ }^{4}$ Rhemautology, Izmir Katip Celebi University School of Medicine: ${ }^{5}$ Nephrology, Izmir Katip Celebi University School of Medicine Ataturk Research Hospital, Izmir, Turkey

Background: Anti-neutrophil cytoplasmic antibody (ANCA)-associated vasculitis (AAV) frequently affect the kidneys and renal involvement is an important factor regarding morbidity and mortality. Kidney lesion in AAV is characterised by necrotizing and crescentic glomerulonephritis by little or no immune deposition, and hence it was called pauci-immune glomerulonephritis (PIGN). The underlying mechanisms in the formation or progression of crescent formation need further investigations. Mammalian target of rapamycin (mTOR) is a serine/threonine kinase and plays role in the regulation of cell growth and proliferation.

Objectives: We aimed to evaluate the role of mTOR, which might be a potential therapeutic target, in kidney biopsies of patients with AAV.

Methods: The patients diagnosed as PIGN at an outpatient nephrology clinics of a tertiary hospital, between May 2009 and June 2016, were retrospectively reviewed and those patients who had a renal biopsy before receiving an immunosuppressive treatment were included in the study. Renal biopsy specimens were immunohistochemically stained with antibodies of mTOR, phosphatase and tensin homolog (PTEN) and transforming growth factor- $\beta$ (TGF- $\beta$ ) and scored by an experienced renal pathologist.

Results: In total 54 patients with AAV ([52\%] female) were included in the study. Twenty-five (46\%) patients were diagnosed as granulomatosis with polyangitis, 6 $(11 \%)$ patients as microscopic polyangiitis, $16(30 \%)$ patients as renal-limited disease, one (2\%) patient as eosinophilic granulomatosis with polyangiitis. Six (11\%) patients with PIGN could not be classified definitely. According to the histopathologic examination; $22 \%$ of the biopsies were classified as focal, $33 \%$ crescentic, $22 \%$ mixed and $22 \%$ as sclerotic. The mTOR was expressed in substantial percentages of glomeruli of patients with PIGN. However we observed PTEN expression in all samples and mTOR in all tubulointerstitial areas. mTOR expression was found to be related with the presence of crescentic and sclerotic changes observed in glomeruli and the degree of fibrosis in interstitial areas. In our study serum creatinine level or response to treatment were not found to be associated with mTOR pathway expression

Conclusions: Our study showed that glomerular or interstitial expression of $\mathrm{PI3K} / \mathrm{Akt} / \mathrm{mTOR}$ pathway may play a role in the pathogenesis of PIGN and mTORC1 inhibitors might provide a viable alternative for this disease.

Disclosure of Interest: None declared

DOI: 10.1136/annrheumdis-2018-eular.1920

\section{THU0459 \\ SURVIVAL OF BIOPSY PROVEN GIANT CELL ARTERITIS IN NORTHERN ITALY: CORRELATION WITH CLINICAL, LABORATORY AND HISTOPATHOLOGICAL} FINDINGS

P. Macchioni ${ }^{1}$, L. Boiardi ${ }^{1}$, F. Muratore ${ }^{1}$, M. Catanoso ${ }^{1}$, A. Cavazza ${ }^{2}$, P. Mancuso ${ }^{3}$, L. Cimino ${ }^{4}$, G. Restuccia ${ }^{1}$, C. Salvarani ${ }^{1} .{ }^{1}$ Rheumatology Unit, ${ }^{2}$ Pathology Unit, ${ }^{3}$ Epidemiology Service; ${ }^{4}$ Ophthalmology Unit, Azienda USL-IRCCS di Reggio Emilia, Reggio Emilia, Italy

Objectives: To correlate survival with clinical, laboratory and histopathological findings in a population based cohort of patients with biopsy-proven giant cell arteritis (GCA) living in the Reggio Emilia area during a 26 years period.

Methods: In this population-based study, all patients living in the Reggio Emilia area who underwent temporal artery biopsy (TAB) for suspected GCA from January 1, 1986 to December 31, 2012 were identified. A pathologist with expertise in vasculitis and blinded to clinical data and final diagnosis reviewed all TABs. Based on the localization of the inflammation, positive TABs were classified into 4 cate gories: small vessel vasculitis (SVV), with inflammation limited to small periadven titial vessels devoid of muscular coat; vasa vasorum vasculitis (VVV), with inflammation surrounding the adventitial vasa vasorum; inflammation limited to adventitia (ILA), with inflammation spreading from vasa vasorum to the adventitia without extension to the media; transmural inflammation (TMI), with external elastic lamina disruption and extension of the inflammation to the media. Histopathologic features evaluated were: the severity of inflammation and intimal hyperplasia, both graded on a semiquantitative scale (mild $=1$, moderate $=2$ severe $=3$ ), the presence of intraluminal acute thrombosis, calcifications, giant cells, fibrinoid necrosis and laminar necrosis. Information about clinical manifestations, laboratory findings, treatment and disease course were collected. Patients were followed from GCA diagnosis to death, migration or December 2013.

Results: 281 patients (206 female, $73.3 \%$ ) with biopsy-proven GCA were identified in the study period. 120 patients ( 84 female, $70 \%$ ) died during a median follow-up period of 96 (IQR 55, 143) months. At univariate analysis, the presence of polymyalgia rheumatica (PMR) (HR 0.54, 95\% Cl 0.37-0.79, $\mathrm{p}=0.002)$, higher level of haemoglobin ( $\mathrm{HR} 0.84,95 \% \mathrm{Cl} 0.74-0.96, \mathrm{p}=0.011$ ) at disease onset, long-term remission ( $\mathrm{HR} 0.47,95 \% \mathrm{Cl} 0.26-0.86, \mathrm{p}=0.015)$ and ILA or VVV at TAB (HR 0.48, 95\% Cl 0.24-0.97, $\mathrm{p}=0.041$ ) were associated with lower mortality, while the evidence of large vessel involvement at imaging studies performed at diagnosis was associated with increased mortality (HR 5.84, 95\% Cl 1.57-21.8 $\mathrm{p}=0.009$ ). Multivariate analysis confirmed the association between lower mortality and PMR (HR 0.54, 95\% Cl 0.36-0.81, p=0.003), higher level of haemoglobin (HR $0.83,95 \% \mathrm{Cl} 0.69-0.99, \mathrm{p}=0.049$ ) at disease onset, and ILA or VVV at TAB ( $\mathrm{HR} 0.38,95 \% \mathrm{Cl} 0.17-0.82, \mathrm{p}=0.014)$, and between increased mortality and large vessel involvement at imaging studies performed at diagnosis (HR 5.31, 95\% Cl 1.39-20.26, $p=0.014$ )

Conclusions: PMR at diagnosis and only adventitial inflammation at TAB seem to identify subsets of biopsy-proven GCA patients with more benign disease, while large vessel involvement at diagnosis a subset with reduced survival.

Disclosure of Interest: None declared

DOI: 10.1136/annrheumdis-2018-eular.6752

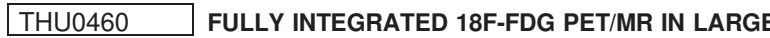 VESSEL VASCULITIS}

R. Padoan ${ }^{1}$, F. Crimì ${ }^{2}$, M. Felicetti ${ }^{1}$, F. Padovano ${ }^{3}$, L. Punzi ${ }^{1}$, C. Lacognata ${ }^{2}$, R. Stramare ${ }^{2}$, D. Cecchin ${ }^{3}$, F. Bui ${ }^{3}$, P. Zucchetta ${ }^{3}$, F. Schiavon ${ }^{1} .{ }^{1}$ Operative Unit of Rheumatology, Department of Medicine DIMED; ${ }^{2}$ Operative Unit of Radiology, Department of Medicine DIMED; ${ }^{3}$ Operative Unit of Nuclear Medicine, Department of Medicine DIMED, University of Padova, Italy

Background: Positron emission tomography (PET) is a non-invasive imaging method that detects ${ }^{18} \mathrm{~F}$-fluorodeoxyglucose (FDG) uptake in vessel's walls. Its simultaneous combination with magnetic resonance (MR) would offer not only a more detailed morphological analysis of the vessels but also a reduction of the radiation, simplifying the clinical workflow and being logistically easier for the patient.

Objectives: To evaluate, for the first time up to now, the usefulness of a fully integrated 18F-FDG PET/MR in a series of large vessels vasculitides (LVV) patients. Methods: We performed a controlled non-randomised prospective study. Images were acquired on a fully integrated PET/MR scanner (Siemens Biograph mMR), consisting in a complete MR protocol and FDG-PET whole body imaging. We evaluated vessel's standard uptake value (SUV) maximum and wall thickness (WT), defined as the mean of 4 measures (at 12, 3, 6 and 9 o'clock) at the inferior margin of D5, D9, D12, L3 and at thickest point (max WT). 